\title{
Effects of Pyramiding Quantitative Resistance Genes pi21, Pi34, and Pi35 on Rice Leaf Blast Disease
}

Nobuko Yasuda, Takayuki Mitsunaga, and Keiko Hayashi, NARO Agricultural Research Center, Tsukuba, Ibaraki 305-8666, Japan; Shinzo Koizumi, NARO Agricultural Research Center, Tsukuba, Ibaraki 305-8666, Japan; and Tsukuba International Center, Japan International Cooperation Agency, Tsukuba, Ibaraki 305-0074, Japan; and Yoshikatsu Fujita, NARO Agricultural Research Center, Tsukuba, Ibaraki 305-8666, Japan; and College of Bioresource Sciences, Nihon University, Fujisawa Kanagawa 252-0880, Japan

\begin{abstract}
Yasuda, N., Mitsunaga, T., Hayashi, K., Koizumi, S., and Fujita, Y. 2015. Effects of pyramiding quantitative resistance genes pi21, Pi34, and Pi35 on rice leaf blast disease. Plant Dis. 99:904-909.

Development of resistant cultivars has been an effective method for controlling rice blast disease caused by Magnaporthe oryzae. Quantitative blast resistance genes may offer durable resistance because the selection pressure on $M$. oryzae to overcome resistance is low as a result of the genes' moderate susceptibility. Because the effects of individual resistance genes are relatively small, pyramiding these genes in rice cultivars is a promising strategy. Here, we used near-isogenic and backcross lines of rice cultivar Koshihikari with single- or two-gene combinations of blast resistance genes (pi21, Pi34, and Pi35) to evaluate the suppression

of leaf blast. The severity of the disease was assessed throughout the infection process. Resistance varied among the lines: Pi35 conferred the strongest resistance, while Pi34 showed the weakest effects. Two types of combined-gene interactions were observed, and they varied on the basis of gene combination and characteristic of the infection: (i) the combination of two resistance genes was more effective than either of the genes individually or (ii) the combination of two resistance genes was similar to the level of the most effective resistance gene in the pair. The most effective gene combination for the suppression of leaf blast was pi21 + Pi35
\end{abstract}

Rice blast caused by Magnaporthe oryzae B.C. Couch \& L.M. Kohn (anamorph, Pyricularia oryzae) is one of the most economically devastating rice diseases worldwide. The use of resistant rice cultivars is an effective way to manage this disease. To date, more than 80 genes for blast resistance have been reported (18). Blast resistance in rice has generally been classified into two categories: qualitative (complete or true) and quantitative (partial or field) resistance $(5,25)$. A majority of the reported blast resistance genes in rice are qualitative resistance genes, which prevent reproduction of the pathogen, and are often associated with a hypersensitive response.

Qualitative resistance genes are race-specific and follow a model of gene-for-gene interaction. Deployment of uniform rice cultivars with simply inherited resistance over vast areas has led to the emergence of new virulent races of blast fungus: this has resulted in the breakdown of cultivar resistance to the disease $(3,16)$, presumably because of the high specificity of the blast resistance genes.

Resistance controlled by quantitative resistance genes allows the formation of sporulating lesions but suppresses lesion expansion or spore formation on rice cultivars, and consequently slows the spread of blast disease. Quantitative resistance genes presumably confer broad-spectrum and durable resistance because the selection pressure on blast fungus to overcome this resistance is low as a result of their moderate susceptibility $(5,21,27)$. Therefore, improving the level of blast resistance in rice using quantitative resistance genes has been a major goal in rice breeding (15).

Recently, intensive genetic analyses of quantitative blast resistance genes including pi21, Pi34, Pi35, Pi39, and Pbl have been conducted $(7,8,10,24,33,39)$, and DNA markers that are tightly

Corresponding author: Nobuko Yasuda, E-mail: yasuda@affrc.go.jp

Accepted for publication 21 October 2014.

http://dx.doi.org/10.1094/PDIS-02-14-0214-RE

(C) 2015 The American Phytopathological Society linked to these resistance genes have been developed (17). Markerassisted selection (MAS) facilitates the selection of rice lines carrying quantitative resistance genes of interest in breeding programs. Since the effects of individual quantitative resistance genes are relatively small, pyramiding of these genes is a useful strategy for further improving resistance to rice blast. However, information on appropriate combinations of resistance genes is largely lacking for this disease.

The effects of pyramiding resistance genes have been investigated for several plant-microbe interactions. In some cases, pyramiding of bacterial blight quantitative resistance genes in rice provided a higher level of resistance than did the introduction of individual genes $(1,29,30,38,40)$. In other cases, pyramiding provided a level of resistance that was comparable to that provided by introducing a single gene in the combination (29). In potatoes, a higher level of resistance against late blight was shown when the resistance genes $\mathrm{R}_{P i-m c d l}$ and $\mathrm{R}_{P i-\text { ber }}$ were pyramided (32). Barley lines containing pyramided quantitative trait loci (QTLs) involved in resistance against stripe rust were found to have increased resistance (26). In a study of three rice blast resistance QTLs ( $q B R 4-2 a$, $q B R 4-2 b$, and $q B R 4-2 c)$, increasing the number of QTLs led to reduced lesion area (6). However, detailed information about the effects of gene pyramiding on the infection process of blast fungus is limited.

In this study, we focused on three quantitative blast resistance genes, pi21, Pi34, and Pi35 in rice. The pi21 gene is recessive and its dominant allele, $P i 21$, encodes a proline-rich protein that includes a putative heavy metal-binding domain and protein-protein interaction motifs (7). Pi34 and Pi35 are the two dominant genes and are located on chromosomes 11 and 1 , respectively $(8,24,39)$. While Pi34 has not yet been isolated, Pi35 was shown to encode a protein containing a nucleotide binding site (NBS) and leucine-rich repeats (LRRs) (8), which are common structures of resistance genes. We used near-isogenic lines (NILs) and backcross lines of the cultivar Koshihikari containing one or two resistance genes (pi21, Pi34, and Pi35), to evaluate the effectiveness of these genes against leaf blast by artificial inoculation. We compared the severity of blast disease in each rice line according to five disease characteristics: (i) penetration frequency of blast fungus; (ii) number of infected rice cells at 
$40 \mathrm{~h}$ after inoculation; (iii) number of sporulating lesions; (iv) lesion length and width; and (v) percentage of diseased leaf area.

\section{Materials and Methods}

Plant materials. The materials used in this study included two NILs containing the blast resistance genes pi21 and Pi34 in the Koshihikari background, a breeding line Chugoku IL1 (23) (called Pi34-IL in this study), and a cultivar Chubu 125 (28) (called pi21-NIL in this study). Chugoku IL1 was developed from the Chugoku 40 breeding line, which carried Pi34. Chugoku 40 was backcrossed six times with Koshihikari, and a line carrying Pi34 was selected by MAS (23). Chubu 125 was developed from a Sensho cultivar that carried the resistant pi21 allele. Sensho was crossed four times with the cultivar Mineasahi, and was then backcrossed twice with Koshihikari, and a progeny carrying pi21 was selected by MAS $(7,28)$. The mother of Mineasahi is Kanto 79, which is a mutant line of Koshihikari $(7,28)$. More than 300 polymorphic genetic markers between parents confirmed that most of the genomic regions of Chubu 125 originated from Koshihikari $(7,28)$.

The rice cultivar Hokkai 188 was used as a donor of the blast resistance gene Pi35 (24). Koshihikari was crossed with Hokkai 188: $\mathrm{F}_{1}$ plants were backcrossed with Koshihikari to develop the $\mathrm{BC}_{1} \mathrm{~F}_{1}$ and the resulting plants were self-pollinated. Individuals that were homozygous for the Pi35 allele were selected from the $\mathrm{BC}_{1} \mathrm{~F}_{2}$ populations by MAS. One selected $\mathrm{BC}_{1} \mathrm{~F}_{2}$ plant was self-pollinated, and the $\mathrm{BC}_{1} \mathrm{~F}_{3}$ plants were designated as Pi35-L. Although the genetic background of Pi35-L differed in part from that of Koshihikari, leaf blast resistance in Pi35-L was assumed to be mainly controlled by Pi35. Pi35 is the only QTL from Hokkai 188 detected in an $\mathrm{F}_{3}$ population derived from a cross between rice cultivar Danghang-Shali, which has poor resistance, and Hokkai188 $(8,24)$. In addition, the susceptibility of Danghang-Shali to leaf blast was comparable to that of Koshihikari; in the field test, the percentage of diseased leaf area was approximately $80 \%$ in Danghang-Shali and Koshihikari and 7\% in Hokkai 188. Thus, Pi35-L could be used to study the effect of Pi35.

Pyramided lines (called pi21 + Pi34-NIL, pi21 + Pi35-L, and Pi34 + $P i 35-\mathrm{L}$ in this study) carrying two blast resistance genes were developed using pi21-NIL, Pi34-IL, and Pi35-L. F 1 plants carrying two resistance genes were self-pollinated and individuals with homozygous alleles at the resistance gene loci were selected from the $\mathrm{F}_{2}$ population using MAS.

$M$. oryzae inoculation and disease scoring. M. oryzae isolate Ao 92-06-2, which is pathogenic to Koshihikari, was used to inoculate Koshihikari and the lines for analysis of resistance gene effects. The method for preparing spore suspensions was the same as described previously (36). Seeds were sown in cell trays containing soil: each cell was $3 \times 3 \times 4.5 \mathrm{~cm}$ and each tray contained 8 rows of 16 cells. Plants were grown in a greenhouse at 20 to $30^{\circ} \mathrm{C}$ under ambient lighting conditions.

The leaf sheath inoculation method (31) was used to investigate the penetration frequency of blast fungus and the number of infected rice cells at $40 \mathrm{~h}$ after inoculation. A sheath of the sixth leaf from the bottom was detached at the 6.5-leaf stage and filled internally with M. oryzae spores $\left(1 \times 10^{4}\right.$ to $3 \times 10^{4} / \mathrm{ml}$ suspended in distilled water) using a syringe. Inoculated leaf sheaths were placed in petri dishes ( $9 \mathrm{~cm}$ diameter) that were kept in a chamber at $25^{\circ} \mathrm{C}$ in darkness. Slices of tissue from the inner surface of the leaf sheaths were removed with a razor blade at $40 \mathrm{~h}$ after inoculation and used for observation by bright-field microscopy. The number of rice cells invaded by infection hyphae was counted on each appressorium. Two independent experiments were performed, each using five sheaths from five plants of each rice line, and 114 appressoria were observed for each sheath.

We used the previously described spray inoculation method (36) to measure the number of sporulating lesions and the percentage of diseased leaf area. A 400-ml sample of $M$. oryzae spore suspension $\left(1 \times 10^{5}\right.$ to $3 \times 10^{5} / \mathrm{ml}$ suspended in distilled water) was sprayed on plants at the 5.8-leaf stage. Three independent experiments were performed, each using 18 to 39 plants for each rice line. The number of sporulating lesions on each plant was counted 6 days after inoculation. At 7 days after inoculation, the sixth leaf from the bottom of each plant was picked and the percentage of diseased leaf area was quantified as previously described (7).
To measure lesion size, we modified the spot inoculation method described by Jia et al. (12). An intact rice plant at the 5.5-leaf stage with roots was placed horizontally in a plastic tray and the sixth leaf from the bottom was inoculated with a 5 - $\mu$ l droplet of spore suspension $\left(5 \times 10^{5}\right.$ to $1 \times 10^{6} \%$ $\mathrm{ml}$ suspended in distilled water containing $0.05 \%$ Tween $20[\mathrm{v} / \mathrm{v}])$. The plant trays were immediately placed in a dark chamber with a moisturesaturated atmosphere at $25^{\circ} \mathrm{C}$ for $20 \mathrm{~h}$ and were then transferred to a greenhouse at $25^{\circ} \mathrm{C}$ under ambient lighting conditions. At 20 days after inoculation, the length and width of each lesion were measured with a caliper (DIGIPA, Mitutoyo, Kawasaki, Japan). Three independent experiments were performed using 10 to 30 plants for each rice line.

Statistical analysis. All data were analyzed using JMP statistical software (version 8.0.1, SAS Institute, Cary, NC). The effect of the presence or absence of the three resistance genes, interactive effects of the genes, and date of inoculation (experimental trial as a random effect) were assessed for each parameter. Penetration status of the blast fungus was assessed using logistic regression analysis. Numbers of infected plant cells at $40 \mathrm{~h}$ after inoculation were compared using Poisson regression analysis to evaluate the effects mentioned above as well as differences among individual plants (nest); when necessary, pairwise comparisons were performed using the Mann-Whitney $U$-test weighted by the Bonferroni correction. Data on numbers, length, and width of lesions were compared using three-way mixed analysis of variance (ANOVA). To satisfy the requirement for normality and homoscedasticity of data, arcsine transformation was conducted on all data for percentage of diseased leaf area, and the transformed data were compared using three-way mixed ANOVA. Where necessary, pairwise comparisons of parametric data were performed using Tukey's honestly significant difference (HSD) test.

\section{Results}

Penetration frequency. The penetration frequency of $M$. oryzae into the epidermal cells of each rice line was evaluated at $40 \mathrm{~h}$ after inoculation and was $>90 \%$ in all lines including Koshihikari. None of the resistance genes (pi21, Pi34, and Pi35) were effective in reducing the penetration frequency of $M$. oryzae (Table 1).

Number of infected rice cells at $40 \mathrm{~h}$ after inoculation. In Koshihikari, approximately 3.9 rice cells were infected at $40 \mathrm{~h}$ after inoculation (Fig. 1), while the numbers of infected cells in lines containing the individual resistance genes pi21, Pi34, and Pi35 were approximately $3.2,1.8$, and 2.3 , respectively (Fig. 1). Poisson regression analysis indicated that Pi34 and Pi35 were significantly effective at reducing the numbers of infected cells at $40 \mathrm{~h}$ after inoculation (Table 2). Pi34 was the most effective of the three genes at reducing the number of infected cells (Fig. 1).

The numbers of infected rice cells in the lines with pi21 + Pi34 was comparable to that in lines with only Pi34, which had fewer infected cells than lines with pi21 (Fig. 1). Similarly, the number of infected cells in the rice lines with pi21 + Pi35 was comparable to that in lines with only Pi35, which had fewer infected cells than lines with only pi21 (Fig. 1). In contrast, lines containing Pi34 + Pi35 showed significantly lower numbers of infected cells than lines containing either Pi34 or Pi35 alone (Fig. 1). Poisson regression analysis revealed a negative interaction between Pi34 and Pi35, which meant that the combined ability of Pi34 + Pi35 to reduce the number of infected rice cells was weaker than the individual effects of these genes (Table 2).

Table 1. Effects of individual resistance genes (pi21, Pi34, and Pi35) and their interactions on the penetration of the Magnaporthe oryzae isolate in comparison with the cultivar Koshihikari, evaluated using logistic regression analysis

\begin{tabular}{lccc}
\hline Sources & df & $\boldsymbol{G}$-value & $\boldsymbol{P}$ value $^{\mathbf{a}}$ \\
\hline pi21 & 1 & 0.48 & 0.49 \\
Pi34 & 1 & 0.08 & 0.78 \\
Pi35 & 1 & 0.00 & 0.98 \\
pi21 × Pi34 & 1 & 0.05 & 0.82 \\
pi21 × Pi35 & 1 & 2.60 & 0.11 \\
Pi34 $\times$ Pi35 & 1 & 2.44 & 0.12 \\
Random effect & 3 & 21.45 & $<0.0001^{*}$ \\
\hline
\end{tabular}

a Asterisk (*) indicates significance $(P<0.05)$. 
Number of sporulating lesions. Each Koshihikari plant contained approximately 85 sporulating lesions (Fig. 2), while the mean number of lesions in lines with pi21, Pi34, and Pi35 were 22, 66, and 37, respectively (Fig. 2). Each of the three resistance genes led to a significant reduction in the number of sporulating lesions (Table 3) and their rank in order of effectiveness was pi21 > Pi35 > Pi34 (Fig. 2).

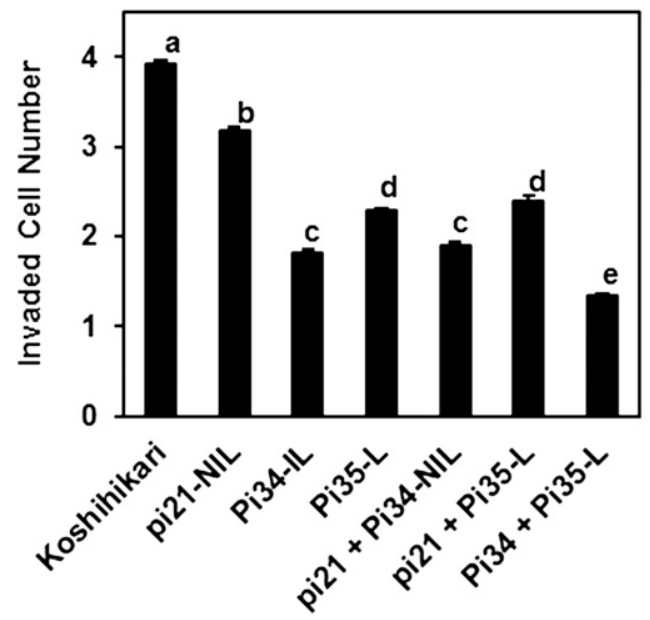

Fig. 1. Number (mean \pm standard error) of rice leaf sheath cells invaded by infection hyphae of Magnaporthe oryzae isolate at $40 \mathrm{~h}$ after inoculation. Values with the same letter are not significantly different (Mann-Whitney U-test with Bonferroni correction, $P>0.05)$. Note that Poisson regression may underestimate the standard error.

Table 2. Effects of individual resistance genes (pi21, Pi34, and Pi35) and their interactions on the number of rice cells infected by the Magnaporthe oryzae isolate in comparison with the cultivar Koshihikari, evaluated using Poisson regression analysis

\begin{tabular}{lrrc}
\hline Sources & df & $\boldsymbol{G}$-value & $\boldsymbol{P}$ value $^{\mathbf{a}}$ \\
\hline pi21 & 1 & 2.17 & 0.1405 \\
Pi34 & 1 & 891.42 & $<0.0001^{*}$ \\
Pi35 & 1 & 175.60 & $<0.0001^{*}$ \\
pi21 × Pi34 & 1 & 58.31 & $<0.0001^{*}$ \\
pi21 × Pi35 & 1 & 92.36 & $<0.0001^{*}$ \\
Pi34 X Pi35 & 1 & 55.02 & $<0.0001^{*}$ \\
Random effect & 3 & 136.19 & $<0.0001^{*}$ \\
Nest & 28 & 134.72 & $<0.0001^{*}$ \\
\hline
\end{tabular}

a Asterisk $(*)$ indicates significance $(P<0.05)$.

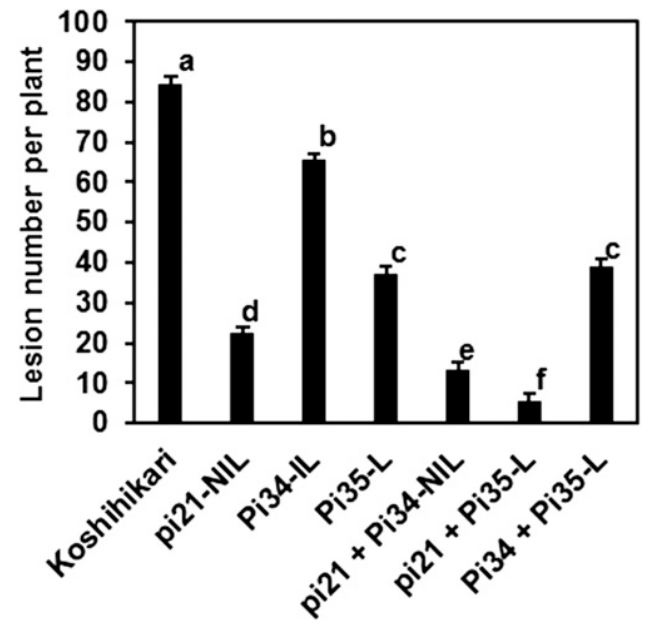

Fig. 2. Numbers of lesions per plant (mean \pm standard error) produced by Magnaporthe oryzae isolate at 6 days after spray inoculation. Values with the same letter are not significantly different (Tukey's honestly significant difference test, $P>$ 0.05).
The number of sporulating lesions in the rice line containing Pi34+ Pi35 was comparable to that in the line containing Pi35 only (Fig. 2). The lines with pi21 + Pi34 or pi21 + Pi35 showed significantly fewer lesions than those with pi21 only (Fig. 2). Negative interactions between resistance genes were detected by the Poisson analysis (Table 3) and indicated that the combined effects of two genes were comparatively smaller than the effects of the individual genes.

Lesion length and width. The shape of lesions on the leaf blades varied with the resistance genes in each rice line (Fig. 3). For example, plants with pi21 formed narrower lesions compared with Koshihikari. Thus, we measured the length and width of sporulating lesions independently.

The mean lesion length in rice lines containing pi21 and Pi34 was comparable to that in Koshihikari (Fig. 4). Rice plants with pi21 had slightly longer lesions than Koshihikari (Fig. 4). The small effect of pi21 on lesion elongation was verified by ANOVA (Table 4). Lesions in rice plants with $P i 35$ were significantly shorter than those in Koshihikari (Fig. 4). The length of lesions in the line with pi21+ Pi34 was comparable to that in the line with pi21 only (Fig. 4), while lesions in lines containing pi21 + Pi35 or Pi34 + Pi35 were comparable in length to those in lines with Pi35 alone (Fig. 4).

Mean lesion width in the rice line with Pi34 was comparable to that in Koshihikari (Fig. 5), while pi21 or Pi35 corresponded to significantly narrower lesions (Table 5). The width of lesions in rice lines with pi21 + Pi34 or Pi34 + Pi35 were comparable to those in rice lines containing the single resistance gene associated with narrower lesions, pi21 and Pi35, respectively (Fig. 5). However, the line with pi21 $+P i 35$ formed narrower lesions than that containing either pi21 or Pi35 alone (Fig. 5), despite detection of a negative interaction between pi21 and Pi35 by ANOVA (Table 5).

Percentage of diseased leaf area. The mean percentage of diseased leaf area in Koshihikari was approximately $60 \%$ (Fig. 6), while diseased leaf area in lines with only pi21, Pi34, or Pi35 was approximately 29,52 , or $15 \%$, respectively (Fig. 6) (all significantly lower than Koshihikari) (Fig. 6). The percentages of diseased leaf area in the rice lines with pi21 + Pi34 were significantly lower than that in the line with pi21 only, which in turn had a lower percentage of diseased leaf area compared with the line with Pi34 only (Fig. 6). Similarly, the percentage of diseased leaf area in the line with pi21 + Pi35 was significantly lower than that in the line with Pi35 only, which showed less diseased area than the line with pi21 only (Fig. 6). However, the percentage of diseased leaf area in the line with Pi34 + Pi35 was comparable to that in the line with Pi35, and these two lines had less diseased leaf area than the line containing Pi34 (Fig. 6). Negative interactions between two of the combined resistance genes were detected, indicating that the effect of these genes in combination was smaller than the sum of the individual effects of the genes (Table 6).

\section{Discussion}

Quantitative blast resistance genes in rice permit the formation of sporulating lesions, but they have the potential to limit proliferation of the disease via specific components of partial resistance. Here, we

Table 3. Effects of individual resistance genes (pi21, Pi34, and Pi35) and their interactions on the number of lesions caused by the Magnaporthe oryzae isolate in comparison with the cultivar Koshihikari, evaluated using three-way mixed analysis of variance (ANOVA)

\begin{tabular}{lrrrr}
\hline Sources & df & \multicolumn{1}{c}{ SS } & F-value & P value \\
\hline pi21 & 1 & 139423.00 & 578.25 & $<0.0001^{*}$ \\
Pi34 & 1 & 1102.08 & 4.57 & 0.032 * $^{*}$ \\
Pi35 & 1 & 37518.70 & 155.61 & $<0.0001^{*}$ \\
pi21 × Pi34 & 1 & 2082.33 & 8.64 & $0.0034^{*}$ \\
pi21 × Pi35 & 1 & 18245.50 & 75.67 & $<0.0001^{*}$ \\
Pi34 × Pi35 & 1 & 9112.29 & 37.79 & $<0.0001^{*}$ \\
Random effect & 2 & 5780.79 & 11.99 & $<0.0001^{*}$ \\
Error & 566 & 136469.25 & & \\
\hline
\end{tabular}

a Asterisk (*) indicates significance $(P<0.05)$. 
found that the development of resistance varied among rice lines containing individual resistance genes. The pi21 gene did not suppress elongation of infection hyphae $40 \mathrm{~h}$ after inoculation but did contribute to a reduction in lesion formation. Fukuoka et al. (7) observed that the rate of invasion of hyphae grown from penetrated cells into adjacent cells $48 \mathrm{~h}$ after inoculation was significantly lower in rice containing pi21. These results suggested that the growth of infection hyphae was reduced later than $40 \mathrm{~h}$ after inoculation, resulting in a decrease in the number of lesions in rice lines with pi21. After the formation of sporulating lesions on rice lines containing pi21, narrow lesions formed that were similar in length to the lesions on Koshihikari. In contrast to pi21, plants containing Pi34 showed strongly reduced elongation of early infection hyphae and slightly suppressed lesion formation. Following the formation of sporulating lesions in rice lines containing Pi34, the length and width of lesions increased to a size that was comparable to those in Koshihikari. Unlike pi21 and Pi34, rice containing Pi35 showed consistently reduced numbers and expansion of lesions. None of the resistance genes had an effect on penetration frequency of the blast fungus.

Two types of combined gene interactions occurred and varied according to the specific gene combination and characteristic of the infection: (i) the combination of two resistance genes was more effective than either of the genes individually; or (ii) the combination of two resistance genes and the most effective of the two resistance genes were similarly effective.

In most cases, rice lines carrying pairs of resistance genes showed disease suppression comparable to that observed in lines containing the gene with the stronger suppressive effect. For example, the number of infected rice cells at $40 \mathrm{~h}$ after inoculation in lines with pi21+ Pi34 was comparable to that in the line with only Pi34 alone, and the number of infected cells in the rice line with pi21 + Pi35 was comparable to that in the line with only Pi35. Moreover, the lesion number and percentage of diseased leaf area in the line with Pi34 + Pi35 were comparable to those in the line with Pi35. In these cases, the behavior of the quantitative resistance genes was similar to that of the qualitative resistance genes reported by Yasuda et al. (37) in that the stronger (or faster-acting) resistance genes were epistatic to the genes with weaker (or slower-acting) effects in rice cultivars containing two resistance genes.

On the other hand, the number of lesions and percentage of diseased leaf area in lines with pi21 + Pi34 and pi21 + Pi35 were significantly lower than those in the lines with the individual resistance genes. The number of infected rice cells at $40 \mathrm{~h}$ after inoculation in the rice line containing Pi34 + Pi35 was less than that in the line with Pi34 alone. We propose the following explanations for the improved suppression of blast disease by pairs of resistance genes compared with the individual genes: (i) in a given tissue, rice plants with two resistance genes may express similar resistance to that shown in plants with one resistance gene; (ii) the member of the gene pair with the stronger effect may be epistatic; (iii) the identity of the epistatic gene may vary according to the type of rice tissue; (iv) the ability of a plant to suppress blast is represented by the total resistance of rice tissues invaded by the fungus; and (v) rice lines carrying pairs of resistance genes may suppress blast disease more effectively than lines carrying a single resistance gene when the epistatic gene varies throughout the infection process. For example, in the rice line

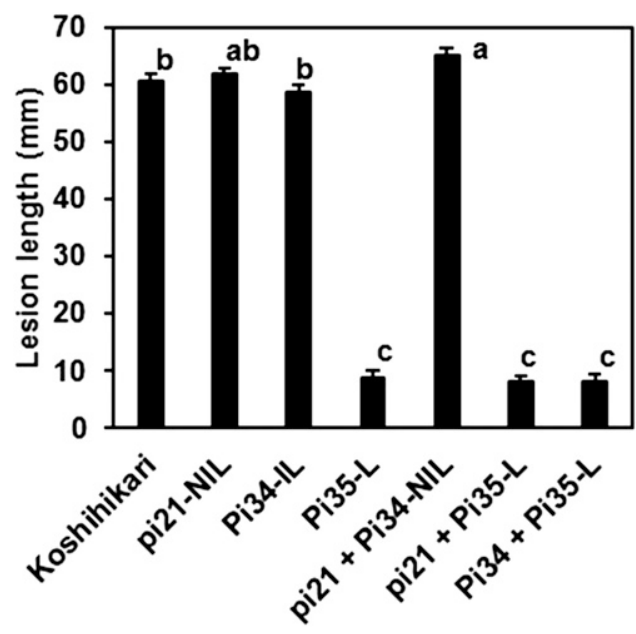

Fig. 4. Lesion length (mean \pm standard error) produced by Magnaporthe oryzae isolate at 20 days after modified spot inoculation. Values with the same letter are not significantly different (Tukey's honestly significant difference test, $P>0.05$ ).

Table 4. Effects of individual resistance genes (pi21, Pi34, and Pi35) and their interactions on the length of lesions caused by the Magnaporthe oryzae isolate in comparison with the cultivar Koshihikari, evaluated using three-way mixed analysis of variance (ANOVA)

\begin{tabular}{lrrrc}
\hline Sources & df & \multicolumn{1}{c}{ SS } & F-value & P value $^{\mathbf{a}}$ \\
\hline pi21 & 1 & 478.04 & 6.12 & $0.0138^{*}$ \\
Pi34 & 1 & 122.87 & 1.57 & 0.2105 \\
Pi35 & 1 & 161802.00 & 2071.22 & $<0.0001^{*}$ \\
pi21 × Pi34 & 1 & 456.83 & 5.85 & $0.016^{*}$ \\
pi21 × Pi35 & 1 & 48.27 & 0.62 & 0.4323 \\
Pi34 × Pi35 & 1 & 33.69 & 0.43 & 0.5117 \\
Random effect & 2 & 93.86 & 0.60 & 0.5489 \\
Error & 424 & 33122.59 & & \\
\hline
\end{tabular}

a Asterisk (*) indicates significance $(P<0.05)$.
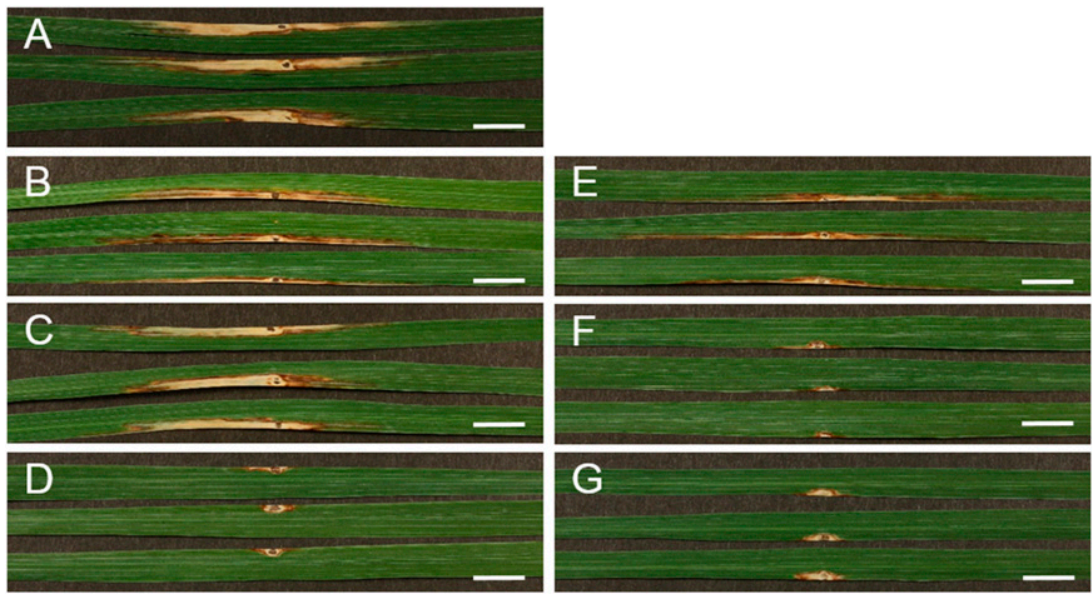

Fig. 3. Lesions produced by Magnaporthe oryzae isolate at 20 days after modified spot inoculation on A, Koshihikari; B, pi21-NIL; C, Pi34-IL; D, Pi35-L; E, pi21 + Pi34-NIL; F, pi21 + Pi35-L; and G, Pi34 + Pi35-L. Scale bars $=1 \mathrm{~cm}$. 
containing pi21 + Pi34, the spread of infection hyphae may be suppressed by Pi34 in epidermal cells, and subsequent elongation of hyphae may be suppressed by pi 21 in mesophyll cells; as a result, these plants had fewer lesions than plants containing pi21 or Pi34 alone. We suggest that $P i 34$ was epistatic to pi21 in epidermal cells, and that pi21 was epistatic to Pi34 in mesophyll cells.

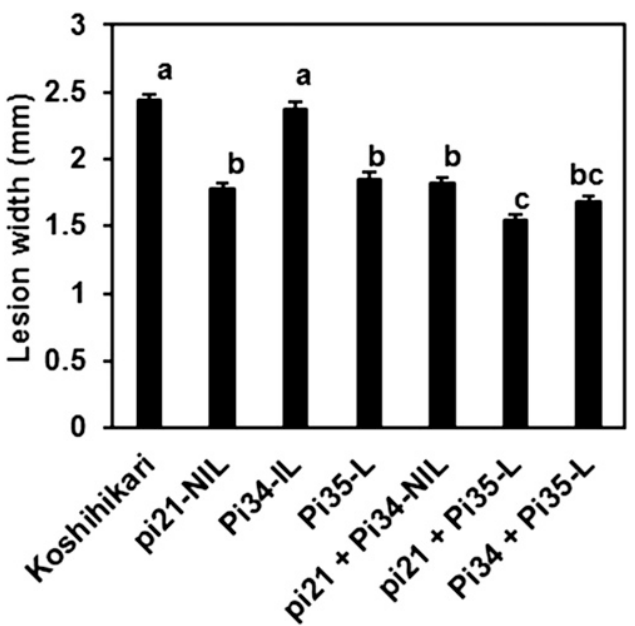

Fig. 5. Lesion width (mean \pm standard error) produced by Magnaporthe oryzae isolate at 20 days after modified spot inoculation. Values with the same letter are not significantly different (Tukey's honestly significant difference test, $P>0.05$ ).

Table 5. Effects of individual resistance genes (pi21, Pi34, and Pi35) and their interactions on the width of lesions caused by the Magnaporthe oryzae isolate in comparison with the cultivar Koshihikari, evaluated using three-way mixed analysis of variance (ANOVA)

\begin{tabular}{lrrrc}
\hline Sources & df & \multicolumn{1}{c}{ SS } & $\boldsymbol{F}$-value & $\boldsymbol{P}$ value \\
\hline pi21 & 1 & 10.11 & 88.60 & $<0.0001^{*}$ \\
Pi34 & 1 & 0.21 & 1.88 & 0.1707 \\
Pi35 & 1 & 12.89 & 112.99 & $<0.0001^{*}$ \\
pi21 $\times$ Pi34 & 1 & 0.20 & 1.75 & 0.1866 \\
pi21 $\times$ Pi35 & 1 & 1.90 & 16.64 & $<0.0001^{*}$ \\
Pi34 $\times$ Pi35 & 1 & 0.13 & 1.13 & 0.2892 \\
Random effect & 2 & 9.92 & 43.46 & $<0.0001^{*}$ \\
Error & 424 & 48.38 & & \\
\hline
\end{tabular}

a Asterisk $(*)$ indicates significance $(P<0.05)$.

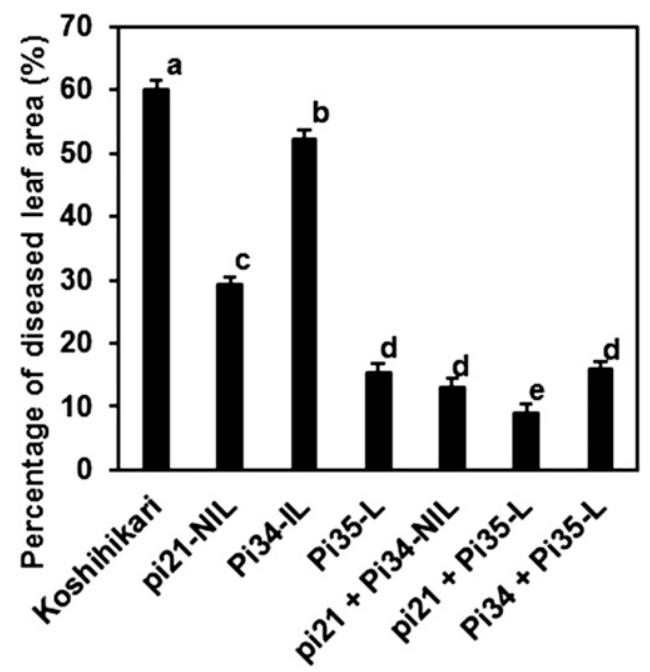

Fig. 6. Percentage of diseased leaf area (mean \pm standard error) infected by Magnaporthe oryzae isolate at 7 days after spray inoculation. Values with the same letter are not significantly different (Tukey's honestly significant difference test, $P>0.05$ ).
In addition to the lesion number, lesion size of the $p 21+P i 35$ rice line was smaller than that of the Pi35 line. This is because lesion width of the pi21 + Pi35 line was narrower than that of the pi21 line, and lesion length was comparable to that of Pi35. The most effective gene combination to decrease the percentage of diseased leaf area was pi $21+$ Pi35.

The pi21 is a recessive gene conferring resistance to blast disease, and its structure differs from those of common resistance genes with NBS-LRR structure, such as Pi35 (8). Nakao et al. (22) demonstrated that the susceptible Pi21 allele of rice enhanced the movement of infection hyphae from penetrated epidermal cells to adjacent mesophyll cells in nonhost Arabidopsis. Thus, the resistance mechanism of pi21 is thought to differ from that of NBS-LRR-type resistance genes. Our results suggested that a combination of genes having different resistance mechanisms, such as pi21 + Pi35, would confer strong resistance effects.

Epistatic effects of resistance gene pairs have been reported in other plant-pathogen interactions. The effector Avr1, from Fusarium oxysporum $\mathrm{f}$. $\mathrm{sp}$. lycopersici, triggers disease resistance in host tomato plants that carry its resistance gene ( $I$ or $I-1)$ in a gene-for-gene manner. However, Avr1 suppresses the effects of other resistance genes (I-2 and I-3) (11). The avrPphC gene of Pseudomonas syringae pv. phaseolicola blocked the avirulence activity of $a v r P p h F$ in the Red Mexican bean cultivar (34). A detailed scenario of epistasis was presented in the Arabidopsis-Pseudomonas interactions. The effector AvrRpt2 from $P$. syringae, which corresponds to the resistance gene RPS2, was found to interfere with AvrB- and AvrRpm1-mediated activation of another resistance gene, RPM1, in Arabidopsis because AvrRpt2 cleaves RIN4, the target protein of AvrB and AvrRpm1 (2,19,20).

Even if there is little interaction among the resistance and effector genes, the highly overlapping signaling network that characterizes resistance might help to explain the epistasis. For example, three resistance genes ( $R P P 4, R P P 7$, and $R P P 8)$ against Peronospora parasitica in Arabidopsis triggered upregulation of common sets of genes, although these three genes differ in their signaling shortly after the recognition event (4). In addition to resistance gene-mediated responses, generally called effector-triggered immunity (ETI), plants express a basal defense of pattern-triggered immunity (PTI) (13). A recent study demonstrated that at least some cases of PTI and ETI depend on common elements of the signaling machinery (9). Katagiri and Tsuda (14) proposed that PTI and ETI result not from differences in the nature of the signaling machinery, but from differences in how the common signaling machinery is used.

In rice- $M$. oryzae interactions, the transcriptional profile at $24 \mathrm{~h}$ after inoculation of $\mathrm{Pil}$-mediated ETI was similar to that of $\mathrm{Pi}$-mediated ETI (35). Moreover, the transcriptional profile of PTI was similar to those of Pil-and Pi9-mediated ETI but the amplitude of changes in gene expression were lower overall (35). These results may suggest PTI and ETI to rice blast also use common signaling machinery in different ways. Katagiri and Tsuda (14) proposed that after perception of a pathogen attack, plants use the common network differently and that different input signals can lead to different mechanisms of signal processing in the network. The epistatic output of the resistance response triggered by resistance gene pairs shown here might be produced

Table 6. Effects of individual resistance genes (pi21, Pi34, and Pi35) and their interactions on the percentage of diseased leaf area caused by the Magnaporthe oryzae isolate in comparison with the cultivar Koshihikari, evaluated using threeway mixed analysis of variance (ANOVA)

\begin{tabular}{lrrrr}
\hline Sources & df & SS & $\boldsymbol{F}$-value & $\boldsymbol{P}_{\text {value }}$ \\
\hline pi21 & 1 & 5.51 & 305.76 & $<0.0001^{*}$ \\
Pi34 & 1 & 0.71 & 39.29 & $<0.0001^{*}$ \\
Pi35 & 1 & 8.27 & 458.69 & $<0.0001^{*}$ \\
pi21 $\times$ Pi34 & 1 & 0.20 & 11.09 & $0.0009^{*}$ \\
pi21 $\times$ Pi35 & 1 & 1.05 & 58.03 & $<0.0001^{*}$ \\
Pi34 $\times$ Pi35 & 1 & 0.17 & 9.57 & $0.0021^{*}$ \\
Random effect & 2 & 0.52 & 14.34 & $<0.0001^{*}$ \\
Error & 550 & 9.92 & & \\
\hline
\end{tabular}

${ }^{a}$ Asterisk $(*)$ indicates significance $(P<0.05)$. 
through signal processing in the common network. Further studies on the molecular mechanisms of individual blast resistance genes will help to clarify positive and negative combinations of resistance genes.

For application to the development of blast-resistant cultivars, the most effective gene combination for reducing the percentage of diseased leaf area was pi21 $+P i 35$. The effectiveness of the combination of pi21 + Pi34 + Pi35 in suppressing blast disease would presumably be comparable to that of pi21+Pi35, because the extent of diseased leaf area in the Pi34 + Pi35 line was comparable to that in the line containing Pi35. However, various factors, including plant age, temperature, insolation, humidity, and agronomic conditions, might influence the effectiveness of resistance genes. Further quantitative studies, implemented under different environmental conditions, should be performed to clarify the mechanisms and effectiveness of gene combinations that confer resistance to rice blast.

\section{Acknowledgments}

This study was supported by grants from the Ministry of Agriculture, Forestry and Fisheries of Japan (Development of new rice varieties using genomic breeding technology, RGB-2004). We thank Dr. Y. Yoshioka (Graduate School of Life and Environmental Sciences, University of Tsukuba, Japan) for kindly providing the software for calculating diseased area and for his helpful advice in data acquisition. We also thank T. Ando and S. Ito (Society for Techno-innovation of Agriculture, Forestry and Fisheries, Japan) for technical cooperation. We thank Dr. H. Maeda (NARO Institute of Crop Science, Japan), Dr. S. Fukuoka (National Institute of Agrobiological Sciences, Japan), and Dr. N. Saka (Mountainous Region Agricultural Research Institute, Aichi Agricultural Research Institute, Japan) for kindly providing the rice line Chugoku IL1 and the rice cultivar Chubu 125, and for their valuable advice.

\section{Literature Cited}

1. Ahmed, H. U., Finckh, M. R., Alfonso, R. F., and Mundt, C. C. 1997. Epidemiological effect of gene deployment strategies on bacterial blight of rice. Phytopathology 87:66-70.

2. Axtell, M. J., and Staskawicz, B. J. 2003. Initiation of RPS2-specified disease resistance in Arabidopsis is coupled to the AvrRpt2-directed elimination of RIN4. Cell 112:369-377.

3. Bonman, J. M. 1992. Durable resistance to rice blast disease-environmental influences. Euphytica 63:115-123.

4. Eulgem, T., Weigman, V. J., Chang, H. S., McDowell, J. M., Holub, E. B., Glazebrook, J., Zhu, T., and Dangl, J. L. 2004. Gene expression signatures from three genetically separable resistance gene signaling pathways for downy mildew resistance. Plant Physiol. 135:1129-1144.

5. Ezuka, A. 1972. Field resistance of rice varieties to rice blast disease. Rev. Plant Prot. Res. 5:1-21.

6. Fukuoka, S., Mizobuchi, R., Saka, N., Ivan, S., Matsumoto, T., Okuno, K., and Yano, M. 2012. A multiple gene complex on rice chromosome 4 is involved in durable resistance to rice blast. Theor. Appl. Genet. 125:551-559.

7. Fukuoka, S., Saka, N., Koga, H., Ono, K., Shimizu, T., Ebana, K., Hayashi, N., Takahashi, A., Hirochika, H., Okuno, K., and Yano, M. 2009. Loss of function of a proline-containing protein confers durable disease resistance in rice. Science 325:998-1001.

8. Fukuoka, S., Yamamoto, S., Mizobuchi, R., Yamanouchi, U., Ono, K., Kitazawa, N., Yasuda, N., Fujita, Y., Nguyen, T. T. T., Koizumi, S., Sugimoto, K., Matsumoto, T., and Yano, M. 2014. Multiple functional polymorphisms in a single disease resistance gene in rice enhance durable resistance to blast. Sci. Rep. 4:4550.

9. Gassmann, W., and Bhattacharjee, S. 2012. Effector-triggered immunity signaling: from gene-for-gene pathways to protein-protein interaction networks. Mol. Plant Microbe Interact. 25:862-868.

10. Hayashi, N., Inoue, H., Kato, T., Funao, T., Shirota, M., Shimizu, T., Kanamori, H., Yamane, H., Hayano-Saito, Y., Matsumoto, T., Yano, M., and Takatsuji, H. 2010. Durable panicle blast-resistance gene $P b 1$ encodes an atypical CC-NBS-LRR protein and was generated by acquiring a promoter through local genome duplication. Plant J. 64:498-510.

11. Houterman, P. M., Cornelissen, B. J., and Rep, M. 2008. Suppression of plant resistance gene-based immunity by a fungal effector. PLoS Pathog. 4: e1000061.

12. Jia, Y., Valent, B., and Lee, F. N. 2003. Determination of host responses to Magnaporthe grisea on detached rice leaves using a spot inoculation method. Plant Dis. 87:129-133.

13. Jones, J. D. G., and Dangl, J. L. 2006. The plant immune system. Nature 444: 323-329.

14. Katagiri, F., and Tsuda, K. 2010. Understanding the plant immune system. Mol. Plant Microbe Interact. 23:1531-1536.

15. Kiyosawa, S. 1981. Gene analysis for blast resistance. Oryza 18:196-203.

16. Kiyosawa, S. 1982. Genetic and epidemiological modeling of breakdown of plant disease resistance. Annu. Rev. Phytopathol. 20:93-117.
17. Koide, Y., Kobayashi, N., Donghe, X. U., and Fukuta, Y. 2009. Resistance genes and selection DNA markers for blast disease in rice (Oryza sativa L.). Jpn. Agric. Res. Q. 43:255-280.

18. Liu, Y., Liu, B., Zhu, X., Yang, J., Bordeos, A., Wang, G., Leach, J. E., and Leung, H. 2013. Fine-mapping and molecular marker development for Pi56(t), a NBS-LRR gene conferring broad-spectrum resistance to Magnaporthe oryzae in rice. Theor. Appl. Genet. 126:985-998.

19. Mackey, D., Belkhadir, Y., Alonso, J. M., Ecker, J. R., and Dangl, J. L. 2003. Arabidopsis RIN4 is a target of the type III virulence effector AvrRpt2 and modulates RPS2-mediated resistance. Cell 112:379-389.

20. Mackey, D., Holt, B. F., 3rd, Wiig, A., and Dangl, J. L. 2002. RIN4 interacts with Pseudomonas syringae type III effector molecules and is required for RPM1-mediated resistance in Arabidopsis. Cell 108, 6:743-754.

21. Miah, G., Rafii, M. Y., Ismail, M. R., Puteh, A. B., Rahim, H. A., Asfaliza, R., and Latif, M. A. 2013. Blast resistance in rice: a review of conventional breeding to molecular approaches. Mol. Biol. Rep. 40:2369-2388.

22. Nakao, M., Nakamura, R., Kita, K., Inukai, R., and Ishikawa, A. 2011. Nonhost resistance to penetration and hyphal growth of Magnaporthe oryzae in Arabidopsis. Sci. Rep. 1:171.

23. National Agriculture and Food Rsearch Organization.Chugoku IL1 Information about breeding lines. Online: http://www.naro.affrc.go.jp/ patent/experiment/cropsystem/cropsystem_kind/rice/007082.html

24. Nguyen, T. T. T., Koizumi, S., La, T. N., Zenbayashi-Sawata, K., Ashizawa, T., Yasuda, N., Imazaki, I., and Miyasaka, A. 2006. Pi35(t), a new gene conferring partial resistance to leaf blast in the rice cultivar Hokkai 188 Theor. Appl. Genet. 113:697-704.

25. Parlevliet, J. E. 1979. Components of resistance that reduce the rate of epidemic development. Annu. Rev. Phytopathol. 17:203-222.

26. Richardson, K. L., Vales, M. I., Kling, J. G., Mundt, C. C., and Hayes, P. M 2006. Pyramiding and dissecting disease resistance QTL to barley stripe rust. Theor. Appl. Genet. 113:485-495.

27. Roumen, E. C. 1994. A strategy for accumulating genes for partial resistance to blast disease in rice within a conventional breeding program. Pages 245-265 in: Rice Blast Disease. R. S. Zeigler, S. Leong, and P. Teng, eds. CAB International, Wallingford, UK/IRRI, Madison, WI.

28. Saka, N., Fukuoka, S., Terashima, T., Kudo, S., Shirota, M., Ando, I., Sugiura K., Sato, H., Maeda, H., Endo, I., Kato, H., and Inoue, M. 2010. Breeding of a new rice variety "Chubu 125 " with high field resistance for blast and excellent eating quality. Res. Bull. Aichi Agric. Res. Ctr. 42:171-183.

29. Singh, S., Sidhu, J. S., Huang, N., Vikal, Y., Li, Z., Brar, D. S., Dhaliwal, H. S., and Khush, G. S. 2001. Pyramidding three bacterial blight resistance genes (xa5, xal3 and $\mathrm{Xa21}$ ) using marker-assisted selection into Indica rice cultivar PR106. Theor. Appl. Genet. 102:1011-1015.

30. Suh, J. P., Jeung, J. U., Noh, T. H., Cho, Y. C., Park, S. H., Park, H. S., Shin, M. S., Kim, C. K., and Jena, K. K. 2013. Development of breeding lines with three pyramided resistance genes that confer broad-spectrum bacterial blight resistance and their molecular analysis in rice. Rice (N Y). Feb 8;6:5.

31. Takahashi, Y. 1958. Disease forecasting of rice blast. Utilization of leaf sheath inoculation assay. Plant Prot. 12:339-345.

32. Tan, M. Y., Hutten, R. C., Visser, R. G., and van Eck, H. J. 2010. The effect of pyramiding Phytophthora infestans resistance genes $\mathrm{R}_{P i-m c d l}$ and $\mathrm{R}_{P i \text {-ber }}$ in potato. Theor. Appl. Genet. 121:117-125.

33. Terashima, T., Fukuoka, S., Saka, N., and Kudo, S. 2008. Mapping of a blast field resistance gene Pi39(t) of elite rice strain Chubu111. Plant Breed. 127: 485-489.

34. Tsiamis, G., Mansfield, J. W., Hockenhull, R., Jackson, R. W., Sesma, A., Athanassopoulos, E., Bennet, M. A., Stevens, C., Vivian, A., Taylor, J. D., and Murillo, J. 2000. Cultivar-specific avirulence and virulence functions assigned to avrPphF in Pseudomonas syringae pv. phaseolicola, the cause of bean halo-blight disease. EMBO J. 19:3204-3214.

35. Wei, T., Ou, B., Li, J., Zhao, Y., Guo, D., Zhu, Y., Chen, Z., Gu, H., Li, C. Qin, G., and Qu, L.-J. 2013. Transcriptional profiling of rice early response to Magnaporthe oryzae identified $O s W R K Y \mathrm{~s}$ as important regulators in rice blast resistance. PLoS ONE 8, 3:e59720.

36. Yasuda, N., Noguchi, M. T., and Fujita, Y. 2005. Identification of an avirulence gene in the fungus Magnaporthe grisea corresponding to a resistance gene at the Pik locus. Phytopathology 95:768-772.

37. Yasuda, N., Noguchi, M. T., and Fujita, Y. 2008. Induced rice resistance to blast varies as a function of Magnaporthe grisea avirulence genes. Plant Dis. 92:1144-1149.

38. Yoshimura, S., Yoshimura, A., Iwata, N., McCouch, S. R., Abenes, M. L., Baraoidan, M. R., Mew, T. W., and Nelson, R. J. 1995. Tagging and combining bacterial blight resistance genes in rice using RAPD and RFLP markers. Mol. Breed. 1:375-387.

39. Zenbayashi-Sawata, K., Fukuoka, S., Katagiri, S., Fujisawa, M., Matsumoto, T., Ashizawa, T., and Koizumi, S. 2007. Genetic and physical mapping of the partial resistance gene, Pi34, to blast in rice. Phytopathology 97 598-602.

40. Zhang, J., Li, X., Jiang, G., Xu, Y., and He, Y. 2006. Pyramiding of Xa7 and $\mathrm{Xa} 21$ for the improvement of disease resistance to bacterial blight in hybrid rice. Plant Breed. 125:600-605. 